\title{
Aliasing Artifact
}

National Cancer Institute

\section{Source}

National Cancer Institute. Aliasing Artifact. NCI Thesaurus. Code C86978.

An artifact that occurs when the scanned body part is larger than field of view (FOV) such

that portions of the object outside of the desired FOV get mapped to an incorrect

location inside the FOV. 